\title{
Sleep and circadian rhythm function and trait impulsivity: An actigraphy study
}

\author{
Niall M. McGowan ${ }^{\mathrm{a}, *}$, Andrew N. Coogan ${ }^{\mathrm{a}}$ \\ a Department of Psychology, Maynooth University, Maynooth, Ireland
}

\section{A R T I C L E I N F O}

\section{Keywords:}

Impulsivity

Actigraphy

Sleep

Circadian rhythm

ADHD

Delayed sleep phase

\begin{abstract}
A B S T R A C T
We report the relationship between daily rest-activity patterns and trait impulsivity in healthy young adults. The Barratt Impulsiveness Scale was used to identify high and low impulsive individuals among a group of 51 volunteers. Participants' sleep behaviour and circadian rhythm function was assessed using week-long actigraphy. High impulsive individuals displayed phase-delayed patterns of sleep, a decreased total sleep time and sleep efficiency, and disrupted circadian function. Such outcomes were also associated with greater self-reported attention deficit hyperactivity disorder symptoms. The results highlight that sleep and circadian rhythm disturbances may be associated with impulsive traits replicating relationships described in psychiatric illnesses in which impulsivity is a core feature.
\end{abstract}

\section{Introduction}

Impulsivity is a multidimensional trait of transdiagnostic importance across several psychiatric conditions. Multiple behavioural aspects are captured by the trait, including patterns of decreased inhibitory control, intolerance towards delayed gratification, non-planning of actions and lack of foresight for negative outcomes (Winstanley et al., 2006; Gullo et al., 2014).

Among the psychiatric syndromes where impulsivity is a core symptom is attention deficit hyperactivity disorder (ADHD), a common condition in both child and adolescent, and adult psychiatry (Fayyad et al., 2007). The core symptomatic features of ADHD are hyperactivity, impulsivity and inattention. For individuals with a diagnosis of ADHD, hyperactive/impulsive symptoms or inattentive may predominate, or the expression may be of a mixed type (Fayyad et al., 2007). Sleep and circadian rhythm disturbances feature prominently in ADHD (Hvolby, 2015; Coogan and McGowan, 2017). Objective and subjective indicators of dysfunctional sleep and insomnia are present in children and adults with the disorder (Cortese et al., 2009; Sobanski et al., 2008; Spruyt and Gozal, 2011), which in turn are associated with worsened symptoms and daytime functioning (Cassoff et al., 2012). Several studies also show a substantial phase delay and attenuated amplitude of circadian parameters indicated by behavioural and physiological rhythms (Baird et al., 2012; Bijlenga et al., 2013; Van Veen et al., 2010) the magnitude of which also associates with worsened clinical outcomes (Baird et al., 2012; Gamble et al., 2013).
It is unclear to what degree sleep and circadian rhythm disturbances in ADHD constitute epiphenomena of the disorder, or might be moderated/mediated by stimulant medications which are the mainstay of treatment (Ironside et al., 2010; Morash-Conway et al., 2017). Studies in at-risk groups absent of psychiatric diagnosis can enable an investigation of sleep and circadian functioning shed of these confounds. As ADHD symptoms are trait-like, rather than representing discrete clinical states, a continuous distribution among the general population has been described where vulnerable individuals occupy the extremes of a normal range of behaviour (Martin et al., 2014; Rodriguez et al., 2007). Instruments which probe for impulsivity and ADHD like traits have previously been deployed in healthy cohorts (Estévez et al., 2014; Kessler et al., 2005; Patton et al., 1995) and can discriminate healthy controls from ADHD and bipolar disorder based on the expression of impulsive traits (Nandagopal et al., 2011).

Support for the previously outlined associations between poor sleep and delayed circadian phase (expressed as later chronotype/eveningness) and impulsivity has been found among the general population using similar instruments (Adan et al., 2010; Caci et al., 2004; Kilgore, 2007; McGowan et al., 2016). Studies using objective measures to describe sleep and circadian activity profiles and associations with impulsivity in non-clinical groups are scarce however. The present study used actigraphy to investigate sleep behaviour and daily rest-activity rhythms in a population of healthy individuals classified into high and low impulsive groups and completing a checklist of ADHD symptoms.

\footnotetext{
* Corresponding author. Current address: Department of Psychiatry, University of Oxford, Warneford Hospital, Oxford OX3 7JX, UK.

E-mail address: niall.mcgowan@psych.ox.ac.uk (N.M. McGowan).
} 


\section{Methods}

\subsection{Participants}

Fifty-four participants were recruited among the general student population attending Maynooth University. Exclusion criteria for participation were: a previous neurological or psychiatric condition, drug or alcohol dependence, autoimmune or endocrine disorder affecting sleep, or sleep disorder. No participants were on current medication for ADHD or mood disorders. Additionally, a total of three individuals were excluded post data collection as a result of actigraph non-compliance $(n=2)$ or technical failure during recording $(n=1)$.The final sample consisted of 51 participants ( 28 female, 23 male) aged $19-34$ years with a mean age of 23.4 years $(S D=3.6)$. Ethical approval for this study was granted by the Biomedical and Life Sciences Committee at Maynooth University and written informed consent obtained from participants.

\subsection{Assessments}

Trait impulsivity was assessed using the Barratt Impulsiveness Scale (BIS-11; Patton et al., 1995) which measures impulsivity according to three domains: "attentional impulsiveness", "motor impulsiveness", and "non-planning impulsiveness". The scale is a self-reported measure consisting of 30 items. Agreement with each statement is endorsed on a four-point Likert type scale ranging from 'Rarely/Never' to 'Almost Always/Always' . Total BIS-11 scores range from 30 - 120. Participants were divided into high and low impulsive groups by median split of total BIS-11 score (median BIS $=62$; high group: $n=25$, mean BIS $=71.1$; low group: $n=26$, mean BIS $=55.7$ ). The median score obtained in the current sample which was used to classify groups closely corresponds with previous normative parameters of the scale (50th percentile BIS $=61$ reported by Malloy-Diniz et al. (2015)).

The Adult ADHD Self-Report Scale (ASRS) six-item screener was used to assess the presence of adult ADHD core symptoms (Kessler et al., 2005). The questionnaire asks participants to rate how each of the items describe how they have felt and conducted themselves over the previous 6 months. The 5-point Likert type scale ranges from 'Never' to 'Very Often' and yields a total score ranging from 0 to 24 with higher scores indicating the presence of ADHD features.

Subjective sleep quality was assessed using the Pittsburgh Sleep Quality Index (PSQI; Buysse et al., 1989) which measures self-reported sleep quality over the past month. The PSQI consists of seven subscale components assessing: sleep quality, sleep latency, sleep duration, habitual sleep efficiency, sleep disturbances, use of sleep medication and daytime dysfunction. Each sub-domain score ranges between 0 and 3 which produces a global PSQI ranging between 0 and 21 with higher scores indicating poorer sleep quality.

The Munich Chronotype Questionnaire (MCTQ) was used to assess chronotype (Roenneberg et al., 2003, 2007). The questionnaire asks respondents how key aspects of their sleep-wake patterns are organised (e.g. bedtime, the amount of time taken to fall asleep, wake time, amount of time taken to get up), which are differentially reported for workdays and for free days. The midsleep on free days, adjusted for accumulated sleep debt (MSF ${ }_{\text {sc }}$ : Roenneberg et al., 2004), was used as a phase marker of entrainment yielding a continuous measure of chronotype. The difference between midsleep on workdays and unadjusted free days was used to determine the social jetlag (SJL) participants accrued over a typical month (Wittmann et al., 2006).

\subsection{Actigraphy monitoring}

The rest-activity patterns of each participant were monitored for a period of seven consecutive days using wrist-worn actigraphs (Actiwatch 2, Philips Respironics, Murraysville, PA) which were worn on the non-dominant hand. The actigraphs used a solid-state piezo- electric accelerometer to passively record movement which were stored in equidistant $15 \mathrm{~s}$ intervals. For the purpose of analysis data were aggregated into 1-min epochs. Participants wore the actigraphs continuously, noting any removals during instances in which the device might become damaged such as during showering or vigorous sport in an accompanying record sheet. Each morning after waking participants completed the Consensus Sleep Diary (CSD; Carney et al., 2012) during the monitoring period to provide a daily record of self-reported bedtime, get-up time, sleep duration, and daytime naps.

\subsection{Data analysis}

Rest-activity patterns for each participant were analysed using Actiware software version 5.57 (Philips Respironics). The software produced actograms for each participant annotated with estimates of individual sleep-wake patterns. Actograms were inspected visually and actigraph removal records and CSD entries were used to verify actigraph concordance, edit the software algorithm parameters, or designate gaps in the activity record as required. The automated scoring algorithm applied computes a weighted value which integrates previous and successive activity counts from epochs adjacent to the epoch that is presently scored. If the sum of these weighted data exceed a defined minimum threshold value the epoch is scored as awake otherwise it is scored as sleep. We selected a medium activity threshold (40 activity counts) which is the software default setting and has previously been validated using PSG and subjective sleep ratings (Kushida et al., 2001). Days with missing actigraph data of more than $3 \mathrm{~h}$ were excluded from further analysis.

The Actiware software was used to estimate the following sleepwake parameters: "Bedtime" and "Get-up Time" defined as the time from when the participant was trying to sleep showing a sustained period of lying still and the time of final awakening respectively; "Time in Bed" defined as the duration between "Bedtime" and "Get-up Time"; "Sleep Onset Latency" defined as the total time between "Bedtime" and sleep onset; "Total Sleep Time" defined as the duration of time between "Time in Bed" and "Get-up Time" scored as sleeping; "Sleep Efficiency" defined as the "Total Sleep Time" expressed as a percentage of "Time in Bed"; "Wakings after Sleep Onset" (WASO) defined as sum of minutes scored as awake occurring between sleep onset and "Get-up Time".

To describe the shape and consistency of the $24 \mathrm{~h}$ rest-activity pattern non-parametric circadian rhythm analysis (NPCRA; Van Someren et al., 1999) was conducted using Visual Basic (VBA). NPCRA produced the following variables: the "interdaily stability" (IS), "intradaily variability" (IV), the mean activity and onset of the most active consecutive $10 \mathrm{~h}$ interval of daily activity ("M10 activity" and "M10 onset", respectively) and of the least active consecutive $5 \mathrm{~h}$ interval of daily activity ("L5 activity" and "L5 onset", respectively), the mean amplitude of the activity rhythm (AMP) and "relative amplitude" of the activity rhythm (RA) normalised for individual differences in total activity. The IS of the rhythm provides a measure for how stable the rhythm is over multiple days indicating how consistent activity patterns are between days. It varies between zero and 1 with higher values supposedly indicating the strength of synchronisation to the environmental zeitgeber. The IV provides information about the fragmentation of the rhythm of rest and activity within the $24 \mathrm{~h}$ day. Higher values indicate a greater frequency of transitions between rest and activity states between all successive hours. The onset time of M10 and L5 provided phase markers of the most active $10 \mathrm{~h}$ period and least active $5 \mathrm{~h}$ period respectively, and the mean hourly activity counts occurring during these periods captured the levels diurnal and nocturnal activity. The amplitude was defined as the difference between M10 activity and L5 activity and the RA produced a measure of amplitude normalised for total activity. RA scores theoretically range from 0 to 1 , with higher values suggesting a more robust circadian rhythm of the rest-activity pattern. To estimate the periodicity of the circadian rest-activity rhythm we calculated the circadian period for each individual over at 
least 6 consecutive days of actigraphy data by fitting $\chi^{2}$ periodograms using the ClockLab Analysis Circadian Toolbox (Actimetrics, Wilmette, IL) for MATLAB R2012b (Mathworks, Natick, MA). The deviation between the period maximum of the rest-activity pattern obtained and the daily period $\mathrm{T}=24$ was used as a measure of pattern variability.

Statistical analyses were performed in SPSS 22 (IBM, Chicago, IL) and in $\mathrm{R}$ version 3.4.2 ( $\mathrm{R}$ Core Team, 2017) using the ppcor package (Kim, 2015). Independent samples $t$-tests or Mann-Whitney U tests were conducted for normally or non-normally distributed data as appropriate to ascertain differences in dependent measures between low impulsive and high impulsive groups. Partial Pearson or Spearman correlations controlling for age and gender were used to explore associations between self-report instruments and actigraphy variables. Data in hh:mm format were converted into decimal time and transposed into a linear format to facilitate meaningful analysis but are reported in original hh:mm format. Regression analysis was via stepwise models. A significance threshold of $p<0.05$ was used for all analyses. Given the exploratory data analysis approach undertaken the results are interpreted unadjusted for multiple comparisons across independent sleep and rest-activity measurements.

\section{Results}

A median split based on global BIS-11 scores was applied to create two impulsivity groups, high and low. The mean global BIS-11 for the low group was 55.7, $\mathrm{SD}=5.2$ and for the high group was 71.1, $\mathrm{SD}=7.2$. For ASRS scores, the mean for the low impulsive group was $8.5, \mathrm{SD}=2.5$ and for the high group the mean was $13.3, \mathrm{SD}=3.7$. Inspection of sample demographics indicated that the low impulsive group (mean age $=23.96, \mathrm{SD}=3.66 ; 16$ females) and the high impulsive group (mean age $=22.72, \mathrm{SD}=3.55 ; 12$ females) did not significantly differ from each other in age $(p=0.225)$ or gender composition $(p=0.331)$. Results of group-wise comparisons of sleep-wake pattern estimates and sleep assessment questionnaires are presented in Table 1. Analysis of actigraphy parameters revealed that the high impulsive group participants had a significantly later bedtime and midsleep than low impulsive group participants. The time in bed and total sleep time were also truncated among the high impulsive group, and sleep was less efficient. No significant differences in self-reported indicators of sleep quality or chronotype and social jetlag were detected between groups.

NPCRA parameters used to describe the shape of daily rest-activity cycles (Table 2) supported sleep timing estimates showing a significantly delayed phase (L5 onset) among the high impulsive group relative to the low impulsive group. M10 activity was elevated in the high impulsive group as was amplitude however there were no significant between group differences for relative amplitude, rhythm stability (IS) and fragmentation (IV), or M10 onset and L5 activity. Across
Table 2

Actigraphy ascertained NPCRA parameters and periodogram results.

\begin{tabular}{|c|c|c|c|c|}
\hline & \multicolumn{2}{|c|}{ Group: mean (SD) or median \pm IQR } & \multicolumn{2}{|c|}{ Statistical difference } \\
\hline & $\begin{array}{l}\text { Low Impulsive } \\
\text { Group, } n=26\end{array}$ & $\begin{array}{l}\text { High Impulsive } \\
\text { Group, } n=25\end{array}$ & $\begin{array}{l}\text { Test } \\
\text { statistic } \\
(d f=1,49)\end{array}$ & $p$ \\
\hline $\begin{array}{l}\text { Interdaily } \\
\text { stability }\end{array}$ & $0.50(0.13)$ & $0.46(0.11)$ & $t=1.421$ & 0.161 \\
\hline $\begin{array}{l}\text { Intradaily } \\
\text { variability }\end{array}$ & $0.82(0.17)$ & $0.80(0.20)$ & $t=0.522$ & 0.604 \\
\hline $\begin{array}{l}\text { Relative } \\
\quad \text { amplitude }\end{array}$ & $0.91 \pm 0.09$ & $0.90 \pm 0.10$ & $U=0.057$ & 0.955 \\
\hline Amplitude & $264.18 \pm 91.29$ & $381.02 \pm 151.95$ & $U=3.203$ & 0.001 \\
\hline $\begin{array}{r}\text { M10 onset: } \\
\text { hh:mm }\end{array}$ & $10: 51(02: 07)$ & $11: 55(02: 18)$ & $t=1.700$ & 0.095 \\
\hline M10 activity & $282.99 \pm 80.64$ & $390.13 \pm 177.64$ & $U=2.939$ & 0.003 \\
\hline $\begin{array}{l}\text { L5 onset: } \\
\text { hh:mm }\end{array}$ & 02:01 (01:16) & $02: 58(01: 33)$ & $t=2.409$ & 0.020 \\
\hline L5 activity & $16.21 \pm 19.07$ & $17.87 \pm 27.30$ & $U=0.810$ & 0.418 \\
\hline Period: hh:mm & $23: 53 \pm 00: 15$ & $23: 48 \pm 00: 27$ & $U=1.047$ & 0.295 \\
\hline $\begin{array}{l}\text { Deviation of } \\
\text { period } \\
\text { from } 24 \mathrm{~h}: \\
\text { hh:mm }\end{array}$ & $00: 09 \pm 00: 22$ & $00: 25 \pm 00: 49$ & $U=2.227$ & 0.026 \\
\hline
\end{tabular}

Significant differences indicated in bold.

the three measures of sleep timing and circadian phase showing significant between group differences it was noted that timing of daily patterns of activity were delayed by approximately $50-60$ mins in the high impulsive group. Representative actograms of each group are shown in Fig. 1. The rhythmic property of the rest-activity profile was estimated using $\chi^{2}$ periodogram analysis (Table 2) revealing participants obtained a period maximum ranging between 22:03 and 26:46 h. The mean length of the estimated period did not significantly differ between groups though the high impulsive group showed a greater period range (Levine's test, two-tailed; $F=4.63, p=0.036$ ). The deviation of period from $24 \mathrm{~h}$ expressed in absolute terms indicated that the magnitude of $\mathrm{T} \neq 24$ was greater in the high impulsive group. Inclusion of gender as a second factor did not produce a significant gender $\times$ impulsive group interaction for any of the dependent variables that were shown above to differ significantly between the low and high impulsive groups.

Within-group correlations among measures controlling for age and gender of participants are reported in Table 3. Significant associations were present between ASRS measures of ADHD like symptom severity and later midsleep, delayed sleep onset, reduced total sleep time, lower sleep efficiency, poorer subjective ratings of sleep, and period variability. Similarly, decomposition of BIS subscales indicated that attentional impulsiveness and non-planning impulsiveness scores were

Table 1

Actigraphy sleep measures and subjective indicators of sleep and chronotype.

\begin{tabular}{|c|c|c|c|c|}
\hline & \multicolumn{2}{|c|}{ Group: mean (SD) or median \pm IQR } & \multicolumn{2}{|l|}{ Statistical difference } \\
\hline Bedtime: hh:mm & 00:24 (01:14) & 01:28 (01:20) & $t=2.960$ & 0.005 \\
\hline Midsleep: hh:mm & 04:32 (01:11) & 05:19 (1:18) & $t=2.250$ & 0.029 \\
\hline Time in bed: hh:mm & 08:54 (00:56) & 08:21 (00:56) & $t=2.063$ & 0.044 \\
\hline Total sleep time: hh:mm & 07:10 (01:20) & 06:22 (01:09) & $t=2.291$ & 0.026 \\
\hline Sleep onset latency: $\min$ & $19.5 \pm 37.64$ & $29.75 \pm 40.09$ & $U=1.413$ & 0.158 \\
\hline PSQI & $5.0 \pm 3.75$ & $6.0 \pm 5.00$ & $U=1.588$ & 0.112 \\
\hline MCTQ MSF $_{\mathrm{sc}}$ : hh:mm & 05:20 (01:23) & 05:44 (01:28) & $t=1.039$ & 0.304 \\
\hline MCTQ SJL: hh:mm & 01:42 (01:03) & $01: 41(00: 59)$ & $t=0.024$ & 0.981 \\
\hline
\end{tabular}

Significant differences indicated in bold. 
Low Impulsive

High Impulsive

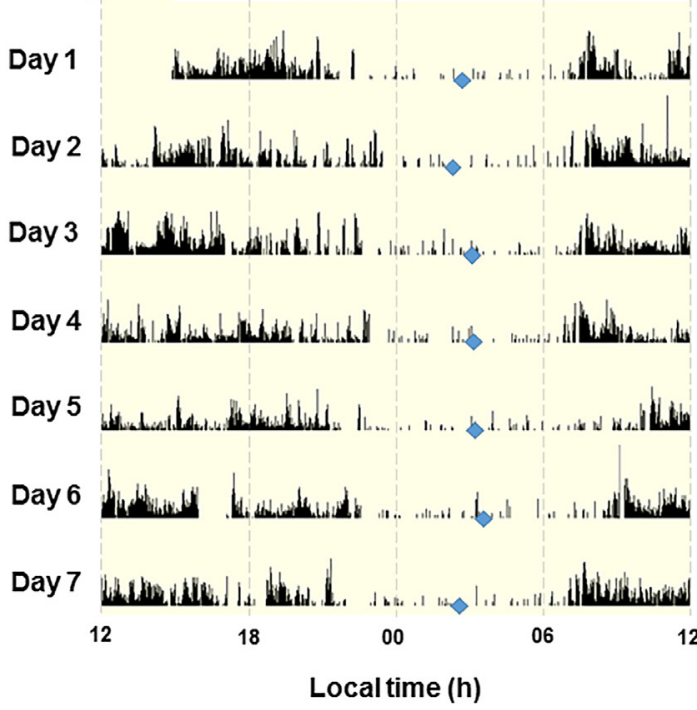

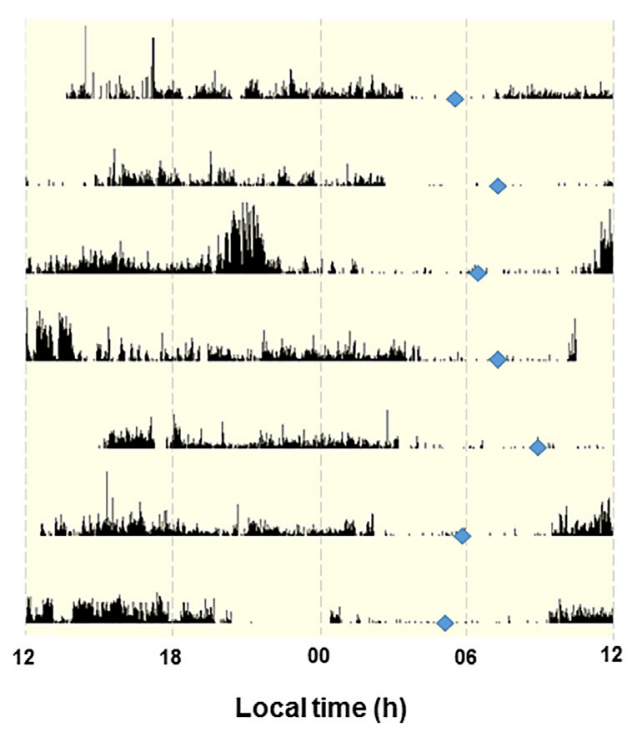

Fig. 1. Sample actograms from two participants, one from the low impulsive group and one from the high impulsive group, recorded for 7 days. Midsleep from each night is indicated by the blue phase markers. Note the markedly later onset of sleep and midsleep phase in the high impulsive actogram compared to the regular sleep onset and earlier sleep phase in the low impulsive actogram. (For interpretation of the references to colour in this figure legend, the reader is referred to the web version of this article.)
Table 3

Partial correlations between ADHD/impulsivity assessments and measures of sleep and circadian function with.

\begin{tabular}{|c|c|c|c|c|c|}
\hline & ASRS & BIS Global & BIS-A & BIS-M & BIS-NP \\
\hline Bedtime & 0.222 & $0.417^{* *}, \dagger$ & $0.484^{* * * *, \dagger}$ & 0.092 & $0.366^{* *, \dagger}$ \\
\hline Get-up time & 0.232 & $0.254^{\dagger}$ & $0.352^{*, \dagger}$ & 0.182 & $0.117^{\dagger}$ \\
\hline Midsleep & $0.312^{*}$ & $0.355^{*}$ & $0.422^{* *, \dagger}$ & 0.214 & $0.228 \dagger$ \\
\hline Time in bed & $-0.091^{+}$ & -0.254 & $-0.228^{\dagger}$ & -0.003 & $-0.346^{*}$ \\
\hline $\begin{array}{l}\text { Total sleep } \\
\text { time }\end{array}$ & $-0.381^{* *}$ & -0.338 & $-0.206^{\dagger}$ & -0.221 & -0.368 \\
\hline $\begin{array}{r}\text { Sleep onset } \\
\text { latency }\end{array}$ & 0.291 & $0.261^{+}$ & 0.042 & 0.238 & 0.184 \\
\hline $\begin{array}{l}\text { Sleep } \\
\quad \text { efficiency }\end{array}$ & $-0.514^{* * *, *}$ & $-0.320^{*}$ & -0.126 & $-0.290^{*}$ & -0.218 \\
\hline WASO & $0.090^{+}$ & $0.091^{*}$ & $0.061^{*}$ & 0.216 & -0.028 \\
\hline PSQI & $0.338^{*}$ & 0.270 & 0.165 & 0.172 & 0.092 \\
\hline MCTQ MSF $_{\mathrm{sc}}$ & $0.135^{+}$ & $0.256^{\dagger}$ & $0.267^{\dagger}$ & $0.148^{+}$ & $0.226^{\dagger}$ \\
\hline MCTQ SJL & 0.039 & $0.106^{\dagger}$ & $0.073^{\dagger}$ & 0.078 & $0.062^{\dagger}$ \\
\hline IS & $-0.221^{+}$ & $-0.209^{\dagger}$ & $-0.129^{\dagger}$ & -0.076 & -0.193 \\
\hline IV & -0.076 & $0.018^{\dagger}$ & $0.114^{\dagger}$ & $-0.081^{+}$ & -0.018 \\
\hline RA & -0.034 & $0.001^{+}$ & -0.010 & -0.027 & 0.156 \\
\hline Amplitude & 0.276 & $0.381^{* * *}$ & 0.287 & 0.136 & $0.428^{* * *+}$ \\
\hline M10 onset & $0.373^{* *+}$ & $0.1511^{\dagger}$ & $0.172 \dagger$ & 0.004 & $0.135^{\dagger}$ \\
\hline M10 activity & 0.274 & $0.355^{*}$ & $0.309^{*}$ & 0.102 & $0.388^{* * *+?}$ \\
\hline L5 onset & 0.189 & $0.245^{\dagger}$ & $0.200^{\dagger}$ & 0.116 & $0.261^{\dagger}$ \\
\hline L5 activity & 0.119 & 0.094 & 0.122 & 0.054 & -0.050 \\
\hline Period & -0.246 & -0.220 & -0.074 & -0.014 & $-0.297^{*}$ \\
\hline $\begin{array}{l}\text { Deviation of } \\
\text { period } \\
\text { from } 24 \mathrm{~h}\end{array}$ & $0.442^{* *,+\%}$ & $0.362^{*}$ & $0.279^{\mathrm{a}, \grave{ }}$ & -0.024 & $0.278^{\mathrm{b}}$ \\
\hline
\end{tabular}

$\dagger$ indicates partial Pearson correlation coefficient.

* indicates partial Spearman correlation coefficient.

${ }^{*} p<0.05$; significant effects highlighted in bold.

*** $p<0.01$; significant effects highlighted in bold.

*** $p<0.001$; significant effects highlighted in bold.

${ }^{\text {a }} p=0.053$.

${ }^{\mathrm{b}} p=0.054$.

associated with late bedtime and midsleep, reduced total sleep time, period variability, and increased diurnal activity, whereas motor impulsiveness only showed modest association with reduced sleep efficiency.

To further analyse the relationships between the sleep and diurnal rhythm variables and global BIS and ASRS scores, variables identified above in the partial correlation analysis as having a statistically significant association with BIS or ASRS scores were entered into a stepwise regression, with BIS or ASRS scores as the dependent variable. For regression onto BIS with the predictor variables of bedtime, midsleep, total sleep time, sleep efficiency, amplitude, M10 activity and deviation of period from $24 \mathrm{~h}$, the maximum variance (adjusted $\mathrm{R}^{2}=0.235$ ) was explained by a model incorporating bedtime $(\beta=0.450, p=0.001)$ and sleep efficiency $(\beta=-0.264, p=0.04)$ as significant predictors. For regression onto ASRS scores, the predictors entered were midsleep, total sleep time, sleep onset latency, sleep efficiency, M10 activity and deviation of period from $24 \mathrm{~h}$. The highest variance accounted for (adjusted $\mathrm{R}^{2}=0.365$ ) resulted from a model incorporating midsleep ( $\beta=0.424, p=0.001)$, PSQI score $(\beta=0.317$, $p=0.111)$ and sleep efficiency $(\beta=-0.266, p=0.29)$. Including interaction terms for gender $\mathrm{x}$ bedtime and gender $\mathrm{x}$ sleep efficiency did not alter the model significantly (Sig. $F$ change $=0.334$ ), indicating that gender did not moderate these relationships. Addition of interaction terms for gender $\mathrm{x}$ midsleep, gender $\mathrm{x}$ PSQI and gender $\mathrm{x}$ sleep efficiency into this model did not increase $\mathrm{R}^{2}$ significantly (Sig. $F$ change $=0.669$ ), indicating that these relationships were not moderated by gender.

\section{Discussion}

This study demonstrated differences in sleep behaviour and the circadian rest-activity structure between individuals expressing high and low levels of trait impulsivity. In accordance with previous reports in ADHD (Coogan and McGowan, 2017), impulsivity in our sample of healthy participants was associated with shorter sleep duration, less efficient sleep, a delayed timing of sleep and greater diurnal arousal, as assessed by actigraphy.

Characterising patterns of sleep disruption and dysfunctional circadian organisation of behaviour may be important in order to understand and treat pathological processes in mental illness (Foster et al., 2013; Wulff et al., 2010). Previous actigraphy studies have focused on quantifying differences in sleep and circadian parameters between different psychiatric diagnostic groups, and compared to healthy groups (Faedda et al., 2016). In a similar manner to studies that show similar disturbances in bipolar disorder and non-clinical groups expressing atrisk symptoms (Ankers and Jones, 2009; Rock et al., 2014), the results of the current study support the hypothesis that sleep which is shorter in duration and delayed in phase is associated with increased impulsivity, as also described in adult ADHD (Fargason et al., 2017; 
Gamble et al., 2013). That core symptoms of psychiatric disorders show associations with specific sleep and circadian rhythm abnormalities in participants without diagnoses suggests that such disturbances may also confer risk among the general population. Elucidation of how these disturbances confer risk for symptom severity will be important to guide future preventative and therapeutic interventions.

Previous studies have indicated associations between later chronotype and eveningness preference with different domains of dysfunctional impulsivity, risk-taking, novelty seeking, and ADHD like symptoms (Adan et al., 2010; Caci et al., 2004; McGowan et al., 2016). It is interesting to note that the current results find that sleep and circadian abnormalities associate more frequently with inattentive and nonplanning aspects of impulsivity, as assessed by the BIS, and a combination of inattentive and impulsive features measured by the ASRS. As impulsivity is not a unitary construct, discrepancies in association may be unsurprising; however, future work using objective methods of monitoring behaviour is required to build a comprehensive understanding of the relation between these disturbances in actual behaviour. The finding that a deviation in the period maximum of the rest-activity rhythm from $24 \mathrm{~h}$ was associated with impulsivity and ADHD like symptoms is in line with previous reports of such associations existing in ADHD (Baird et al., 2012), and in suicidal patients with borderline and impulsive personality traits (Verkes et al., 1996). Under usual circumstances, the circadian clock is entrained to the daily $24 \mathrm{~h}$ period which maintains the appropriate timing of physiological and behavioural functions throughout the day. Non-entrained conditions resulting in free-running of the circadian rhythm are rare outside of blind individuals (Uchiyama and Lockley, 2015) or profound psychotic state (Wulff et al., 2012); however in healthy participants exposure to light pulses in the evening can entrain the pacemaker to non-24h days (Gronfier et al., 2007). The interval recorded in this study only gives a snapshot of the rest-activity period over one week and most likely captures between day variability in the circadian phase of entrainment rather than an abnormal rhythmic component. Delays in bedtime, midsleep and wake time may also be indicative of an altered phase of entrainment in those with higher impulsivity and/or more ADHD like symptoms. Data from adults with ADHD indicate that delayed phase of entrainment is a common finding (Coogan and McGowan, 2017), and these findings may generalise to impulsivity in non-clinical populations. Together with the suggestion of a delayed rest-activity pattern, future studies might better understand impulsivity and the entrainment properties of the circadian clock by monitoring validated phase-markers such as core body temperature and dim-light melatonin onset (Benloucif et al., 2005).

The current study has a number of limitations, including the use of self-report scales for measuring ADHD symptoms and impulsivity by which participants were grouped and the cross-sectional nature of our data which preclude the direction of effect from being ascertained. First, through the use of self-report measures, the results are somewhat dependent on participants' insight into their own behaviour and accuracy in reporting such. This may be problematic as subjective measurement of impulsivity often does not reflect objective measures of behaviour and thus further investigation using neuropsychological tasks which probe such would be beneficial. Second, we cannot determine directionality or causality from the associations described; the interaction between sleep and circadian disturbances and symptom expression consists of several overlapping factors as well as feedforward and feedback drives (Foster et al., 2013). Such factors may include greater levels of arousal (Gruber et al., 2012), sleep deprivation and social jetlag (McGowan et al., 2016), and the moderating role of personality differences (McGowan et al., 2017; Micic et al., 2017; Wilhelmsen-Langeland et al., 2014). Another caveat is using a median split to dichotomize the BIS measurement into low and high impulsivity groups. Such approaches may result in a loss of statistical power and fidelity (Altman and Royston, 2006). We aimed to partially address this issue by including multiple regression analysis based on the continuous
BIS and ASRS variables to recapitulate the findings from the group-wise analysis. A final observation is that this study was observational and exploratory in nature, and as such the findings of the study should be viewed as hypothesis-generating and a prompt for future study (Shrout and Rodgers, 2018).

\section{Conclusions}

Alterations in diurnal profiles of activity and sleep, and decreased sleep quality, were recorded in high impulsive relative to low impulsive individuals, and such disturbances were associated with worsened ADHD like traits. The replication of well described sleep phenotypes in ADHD among a sample of healthy young adults speaks to the transdiagnostic relevance of sleep and circadian rhythm disturbances in conditions where impulsivity is expressed.

\section{Conflicts of interest}

None

\section{Acknowledgements}

Funding: This work was supported by the John and Pat Hume Scholarship Scheme at Maynooth University awarded to NMMcG

\section{References}

Adan, A., Natale, V., Caci, H., Prat, G., 2010. Relationship between circadian typology and functional and dysfunctional impulsivity. Chronobiol. Int. 27 (3), 606-619.

Altman, D.G., Rosyston, P., 2006. The cost of dichotomising continuous variables. BMJ 332 (7549), 1080.

Ankers, D., Jones, S.H., 2009. Objective assessment of circadian activity and sleep patterns in individuals at behavioural risk of hypomania. J. Clin. Psychol. 65 (10), 1071-1086.

Baird, A.L., Coogan, A.N., Siddiqui, A., Donev, R.M., Thome, J., 2012. Adult attention deficit hyperactivity disorder is associated with alterations in circadian rhythms at the behavioural, endocrine and molecular levels. Mol. Psychiatry. 17 (10), 988-995.

Benloucif, S., Guico, M.J., Reid, K.J., Wolfe, L.F., L'Hermite-Balériaux, M., Zee, P.C., 2005 Stability of melatonin and temperature as circadian phase markers and their relation to sleep times in humans. J. Biol. Rhythms. 20 (2), 178-188.

Bijlenga, D., Van Someren, E.J., Gruber, R., Bron, T.I., Kruithof, I.F., Spanbroek, E.C., et al., 2013. Body temperature, activity and melatonin profiles in adults with attention-deficit/hyperactivity disorder and delayed sleep: a case-control study. J. Sleep Res. 22 (6), 607-616.

Buysse, D.J., Reynolds, C.F., Monk, T.H., Berman, S.R., Kupfer, D.J., 1989. The Pittsburgh Sleep Quality Index: a new instrument for psychiatric practice and research. Psychiatry Res. 28 (2), 193-213.

Caci, H., Robert, P., Boyer, P., 2004. Novelty seekers and impulsive subjects are low in morningness. Eur. Psychiatry. 19, 79-84.

Carney, C.E., Buysse, D.J., Ancoli-Israel, S., Edinger, J.D., Krystal, A.D., Lichstein, K.L., et al., 2012. The consensus sleep diary: standardizing prospective sleep self-monitoring. Sleep 35 (2), 287-302.

Cassoff, J., Wiebe, S.T., Gruber, R., 2012. Sleep patterns and the risk for ADHD: a review. Nat. Sci. Sleep. 4, 73-80.

Coogan, A.N., McGowan, N.M., 2017. A systematic review of circadian function, chronotype and chronotherapy in attention deficit hyperactivity disorder. ADHD Atten. Def. Hyp. Disord. 9 (3), 129-147.

Cortese, S., Faraone, S.V., Konofal, E., Lecendreux, M., 2009. Sleep in children with attention-deficit/hyperactivity disorder: meta-analysis of subjective and objective studies. J. Am. Acad. Child Adolesc. Psychiatry. 48(9), 894-908.

Estévez, N., Eich-Höchli, D., Dey, M., Gmel, G., Studer, J., Mohler-Kuo, M., 2014. Prevalence of and associated factors for adult attention deficit hyperactivity disorder in young Swiss Men. PLoS ONE 9 (2), e89298.

Faedda, G.L., Ohashi, K., Hernandez, M., McGreenery, C.E., Grant, M.C., Baroni, A., et al., 2016. Actigraph measures discriminate pediatric bipolar disorder from attentiondeficit/hyperactivity disorder and typically-developing controls. J. Child Psychol. Psychiatry 57 (6), 706-716.

Fargason, R.E., Fobian, A.D., Hablitz, L.M., Paul, J.R., White, B.A., Cropsey, K.L., et al., 2017. Correcting delayed circadian phase with bright light therapy predicts improvement in ADHD symptoms: a pilot study. J. Psychiatr. Res. 91, 105-110.

Fayyad, J., de Graaf, R., Kessler, R., Alonso, J., Angermeyer, M., Demyttenaere, K., et al., 2007. Cross-national prevalence and correlates of adult attention-deficit hyperactivity disorder. Br. J. Psychiatry. 190 (5), 402-409.

Foster, R.G., Peirson, S.N., Wulff, K., Winnebeck, E., Vetter, C., Roenneberg, T., 2013. Sleep and circadian rhythm disruption in social jetlag and mental illness. Prog. Mol. Biol. Transl. Sci. 119, 325-346.

Gamble, K.L., May, R.S., Besing, R.C., Tankersly, A.P., Fargason, R.E., 2013. Delayed sleep 
timing and symptoms in adults with attention-deficit/hyperactivity disorder: a controlled actigraphy study. Chronobiol. Int. 30 (4), 598-606.

Gronfier, C., Wright, K.P., Kronauer, R.E., Czeisler, C.A., 2007. Entrainment of the human circadian pacemaker to longer-than-24-h days. PNAS 104 (21), 9081-9086.

Gruber, R., Fontil, L., Bergmame, L., Wiebe, S.T., Amsel, R., Frenette, S., et al., 2012. Contributions of circadian tendencies and behavioral problems to sleep onset problems of children with ADHD. BMC Psychiatry. 12 (1), 212.

Gullo, M.J., Loxton, N.J., Dawe, S., 2014. Impulsivity: Four ways five factors are not basic to addiction. Addict. Behav. 39 (11), 1547-1556.

Hvolby, A., 2015. Associations of sleep disturbance with ADHD: implications for treatment. ADHD Atten. Def. Hyp. Disord. 7 (1), 1-18.

Ironside, S., Davidson, F., Corkum, P, 2010. Circadian motor activity affected by stimulant medication in children with attention-deficit/hyperactivity disorder. J. Sleep Res. 19 (4), 546-551.

Kessler, R.C., Adler, L., Ames, M., Demler, O., Faraone, S., Hiripi, E., et al., 2005. The world health organization adult ADHD self-report scale (ASRS). Psychol. Med. 35, 245-256.

Kilgore, W.D., 2007. Effects of sleep deprivation and morningness-eveningness traits on risk taking. Psychol. Rep. 100 (2), 613-626.

Kim, S., 2015. Ppcor: an R package for a fast calculation to semi-partial correlation coefficients. CSAM 22 (6), 665-674.

Kushida, C.A., Chang, A., Gadkary, C., Guilleminault, C., Carrillo, O., Dement, W.C., 2001. Comparison of actigraphic, polysomnographic, and subjective assessment of sleep parameters in sleep-disordered patients. Sleep Med. 2, 389-396.

Malloy-Diniz, L.F., Paula, J.J.D., Vasconcelos, A.G., Almondes, K.M.D., Pessoa, R., Faria, L., et al., 2015. Normative data of the Barratt Impulsiveness Scale 11 (BIS-11) for Brazilian adults. Rev. Bras. Psiquiatr. 37 (3), 245-248.

Martin, J., Hamshere, M.L., Stergiakouli, E., O'Donovan, M.C., Thapar, A., 2014. Genetic risk for attention-deficit/hyperactivity disorder contributes to neurodevelopmental traits in the general population. Biol. Psychiatry. 76 (8), 664-671.

McGowan, N.M., Brannigan, R., Doyle, D., Coogan, A.N., 2017. Diurnal preference, circadian phase of entrainment and time perspectives: Just what are the relationships? Pers. Indiv. Dif. 112, 79-84.

McGowan, N.M., Voinescu, B.I., Coogan, A.N., 2016. Sleep quality, chronotype and social jetlag differentially associate with symptoms of attention deficit hyperactivity disorder in adults. Chronobiol. Int. 33 (10), 1433-1443.

Micic, G., Lovato, N., Gradisar, M., Lack, L.C., 2017. Personality differences in patients with delayed sleep-wake phase disorder and non-24-h sleep-wake rhythm disorder relative to healthy sleepers. Sleep Med. 30, 128-135.

Morash-Conway, J., Gendron, M., Corkum, P., 2017. The role of sleep quality and quantity in moderating the effectiveness of medication in the treatment of children with ADHD. ADHD Atten. Def. Hyp. Disord. 9 (1), 31-38.

Nandagopal, J.J., Fleck, D.E., Adler, C.M., Mills, N.P., Strakowski, S.M., DelBello, M.P., 2011. Impulsivity in adolescents with bipolar disorder and/or attention-deficit/hyperactivity disorder and healthy controls as measured by the Barratt Impulsiveness Scale. J. Child. Adolesc. Psychopharmacol. 21 (5), 465-468.
Patton, J.H., Stanford, M.S., Barratt, E.S., 1995. Factor structure of the Barratt Impulsiveness Scale. J. Clin. Psychol. 51, 768-774.

Rock, P., Goodwin, G., Harmer, C., Wulff, K., 2014. Daily rest-activity patterns in the bipolar phenotype: a controlled actigraphy study. Chronobiol. Int. 31 (2), 290-296.

Rodriguez, A., Järvelin, M.-R., Obel, C., Taanila, A., Miettunen, J., Moilanen, I., 2007. Do Inattention and Hyperactivity Symptoms Equal Scholastic Impairment? Evidence from Three European Cohorts 7. BMC Public Health, pp. 327.

Roenneberg, T., Kuehnle, T., Juda, M., Kantermann, T., Allebrandt, K., Gordijn, M., et al., 2007. Epidemiology of the human circadian clock. Sleep Med. Rev. 11, 429-438.

Roenneberg, T., Kuehnle, T., Pramstaller, P.P., Ricken, J., Havel, M., Guth, A., et al., 2004. A marker for the end of adolescence. Curr. Biol. 14 (24), R1038-R1039.

Roenneberg, T., Wirz-Justice, A., Merrow, M., 2003. Life between clocks: daily temporal patterns of human chronotypes. J. Biol. Rhythms 18 (1), 80-90.

Shrout, P., Rodger, J.L., 2018. Psychology, science, and knowledge construction: broadening perspectives from the replication crisis. Annual Rev. Psychol. 69, 487-510.

Sobanski, E., Schredl, M., Kettler, N., Alm, B., 2008. Sleep in adults with attention deficit hyperactivity disorder (ADHD) before and during treatment with methylphenidate: a controlled polysomnographic study. Sleep 31 (3), 375-381.

Spruyt, K., Gozal, D., 2011. Sleep disturbances in children with attention-deficit/hyperactivity disorder. Expert Rev. Neurother. 11 (4), 565-577.

Uchiyama, M., Lockley, S.W., 2015. Non-24-hour sleep-wake rhythm disorder in sighted and blind patients. Sleep Med. Clin. 10 (4), 495-516.

Van Someren, E.J., Swaab, D.F., Colenda, C.C., Cohen, W., McCall, W.V., Rosenquist, P.B., 1999. Bright light therapy: improved sensitivity to its effects on rest-activity rhythms in Alzheimer patients by application of nonparametric methods. Chronobiol. Int. 16 (4), 505-518.

Van Veen, M.M., Kooij, J.J., Boonstra, A.M., Gordijn, M.C., Van Someren, E.J., 2010. Delayed circadian rhythm in adults with attention-deficit/hyperactivity disorder and chronic sleep-onset insomnia. Biol. Psychiatry. 67 (11), 1091-1096.

Verkes, R.J., Kerkhof, G.A., Beld, E., Hengeveld, M.W., Kernpen, G.M.J., 1996. Suicidality, circadian activity rhythms and platelet serotonergic measures in patients with recurrent suicidal behaviour. Acta Psychiatr. Scand. 93 (1), 27-34.

Wilhelmsen-Langeland, A., Saxvig, I.W., Pallesen, S., Nordhus, I.H., Vedaa, Ø., Sørensen, E., et al., 2014. The personality profile of young adults with delayed sleep phase disorder. Behav. Sleep Med. 12 (6), 481-492.

Winstanley, C.A., Eagle, D.M., Robbins, T.W., 2006. Behavioural models of impulsivity in relation to ADHD: translation between clinical and preclinical studies. Clin. Psychol. Rev. 26, 379-395.

Wittmann, M., Dinich, J., Merrow, M., Roenneberg, T., 2006. Social jetlag: misalignment of biological and social time. Chronobiol. Int. 23 (1-2), 497-509.

Wulff, K., Dijk, D.-J., Middleton, B., Foster, R.G., Joyce, E.M., 2012. Sleep and circadian rhythm disruption in schizophrenia. Br. J. Psychiatry. 200 (4), 308-316.

Wulff, K., Gatti, S., Wettstein, J.G., Foster, R.G., 2010. Sleep and circadian rhythm disruption in psychiatric and neurodegenerative disease. Nat. Rev. Neurosci. 11 (8), 589-599. 